\title{
Studies of Non-Proportionality in Alkali Halide and Strontium Iodide Scintillators Using SLYNCI
}

Larry Ahle ${ }^{1}$, Gregory Bizarri ${ }^{2}$, Lynn Boatner ${ }^{3}$, Nerine J. Cherepy ${ }^{1}$, Woon-Seng Choong ${ }^{2}$, William W. Moses ${ }^{2}$, Stephen A. Payne ${ }^{1}$, Kanai Shah ${ }^{4}$, Steven Sheets ${ }^{1}$, and Benjamin, W. Sturm ${ }^{1}$

${ }^{1}$ Lawrence Livermore National Laboratory, 7000 East Avenue, Livermore, CA 94551, U.S.A.

${ }^{2}$ Lawrence Berkeley National Laboratory, 1 Cyclotron Road, Berkeley, CA 94720, U.S.A.

${ }^{3}$ Oak Ridge National Laboratory, Oak Ridge, TN 37831

${ }^{4}$ Radiation Monitoring Devices, Watertown, MA 02472

\begin{abstract}
Recently a collaboration of LLNL and LBNL has constructed a second generation Compton coincidence instrument to study the non-proportionality of scintillators [1-3]. This device, known as SLYNCI (Scintillator Light-Yield Non-proportionality Characterization Instrument), has can completely characterize a sample with less than 24 hours of running time. Thus, SLYNCI enables a number of systematic studies of scintillators since many samples can be processed in a reasonable length of time. These studies include differences in nonproportionality between different types of scintillators, different members of the same family of scintillators, and impact of different doping levels. The results of such recent studies are presented here, including a study of various alkali halides, and the impact of europium doping level in strontium iodide. Directions of future work area also discussed.
\end{abstract}

\section{INTRODUCTION}

The achievable energy resolution for a given scintillator material used for gamma ray spectroscopy is principally dependent on the efficiency for conversion of the electrons and holes into scintillation light, or light yield. The efficiency for this process is not independent of energy deposited in the crystal which leads to a non-proportionality between energy deposited in the crystal and the light yield produced. This non-proportionality is often the limiting factor in determining the final resolution of the crystal [4-7]. To measure this proportionality, a collaboration of LLNL and LBNL has constructed a second generation Compton coincidence instrument known as SLYNCI (Scintillator Light-Yield Non-proportionality Characterization Instrument) [1-3]. This instrument has over a 30 times higher data collection rate than previous devices,[7-9] enabling a complete non-proportionality measurement in less than 24 hours. This increase throughput makes it more straight forward to make systematic studies of scintillator and non-proportionality. These studies include differences in non-proportionality between different types of scintillators, different members of the same family of scintillators, and impact of different doping levels. Such studies were performed for several alkali halides, strontium iodide, and lanthanum bromide.

\section{EXPERIMENT}

SLYNCI relies on Compton scattering events to measure the non-proportionality of a scintillator. It consists of a collimated ${ }^{137} \mathrm{Cs}$ source, a PMT for light detection from the 
scintillator being tested, and five HPGe detectors surrounding the scintillator. Gamma rays with energy of $661.660 \mathrm{keV}$ from the ${ }^{137} \mathrm{Cs}$ source are Compton scattered in the scintillator with the scattered photon being detected in one of the HPGe. The energy measured in the HPGe detector then determines how much energy was deposited in the scintillator, while the signal from the PMT measures how much light was produced by the scintillator. With this information for the entire range of Compton electron energies, the non-proportionality curve can be determined.

To cover the entire energy range, two data runs are taken, one with collimator at $0^{\circ}$ and one at $15^{\circ}$ relative to the center of the middle HPGe detector. The signals from all detectors are fed through charge sensitive preamps and then into $100 \mathrm{MHz}$ waveform digitizers from Struck. A separate FPGA takes the trigger output from the digitizers and processes for coincidence events between the PMT and one of the HPGe. Scaled down singles events from the PMT and the HPGe detectors are also mixed into the data stream for calibration purchases. The PMT and HPGe that sees low angle Compton scattered events are calibrated every roughly every 10 minutes. The other four HPGe are calibrated from a separate calibration run. Signals in valid events are then processed online through a trapezoidal filter to generate signal amplitudes for each triggered detector. Additionally, the raw waveform from the PMT is saved to allow for processing offline.

\section{$\underline{\text { Alkali Halide Data }}$}

Several alkali halides were run through SLYNCI and the data is shown in figure 1. The $\mathrm{NaI}(\mathrm{Tl})$ crystal was manufactured by Saint Gobain and is a cylinder with diameter of $12.5 \mathrm{~mm}$ and length of $12.5 \mathrm{~mm}$. The CsI(Tl) crystal was also manufactured by Saint Gobain but has a diameter of $20 \mathrm{~mm}$ and length of $20 \mathrm{~mm}$. The CsI(Na) crystal was manufactured by ScintiTech and is a cylinder with a diameter of $10 \mathrm{~mm}$ and length of $10 \mathrm{~mm}$. All three curves show somewhat similar behavior but note $\mathrm{NaI}(\mathrm{Tl})$ has a peak at slightly higher energy and at a lower value, giving $\mathrm{NaI}(\mathrm{Tl})$ a slightly better overall non-porportionality.

\section{$\underline{\text { Strontium Iodide Data }}$}

We have begun to study strontium iodide specifically looking at effects due to europium dopant concentration. Figure 2 shows the relative yield for one $\operatorname{SrI}_{2}(\mathrm{Eu})$ crystal grown at ORNL. The dopant concentration is $6 \%$ as tests at LLNL have shown this concentration appears to maximize the light yield. The shape of the $\mathrm{SrI}_{2}$ curve is similar to the alkali halide curves but the amplitude change is significantly less. Specifically, the peak value for the alkali halides is about $15-25 \%$ as compared to their value at $446 \mathrm{keV}$. For the $\mathrm{SrI}_{2}$ this change is only about $5 \%$. The increased scatter seen in this data is believed to be due to energy escaping from the $\mathrm{SrI}_{2}$ crystal as this crystal was a cube of roughly $5 \mathrm{~mm}$ a side. 


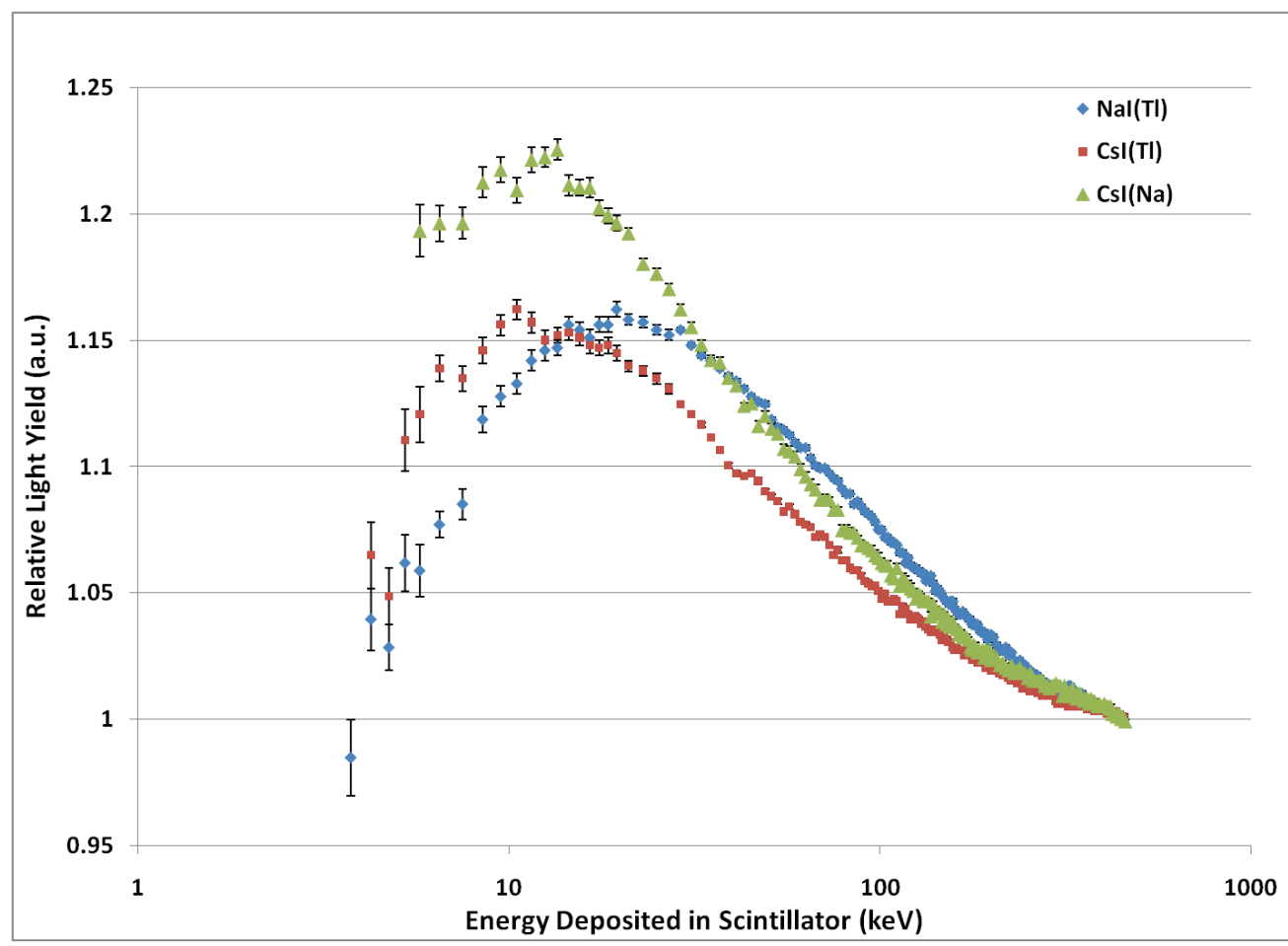

Figure 1. Relative light yield curves for $\mathrm{NaI}(\mathrm{Tl}), \mathrm{CsI}(\mathrm{Tl})$ and $\mathrm{CsI}(\mathrm{Na})$. Each curve has been renormalized to its value at $446 \mathrm{keV}$.

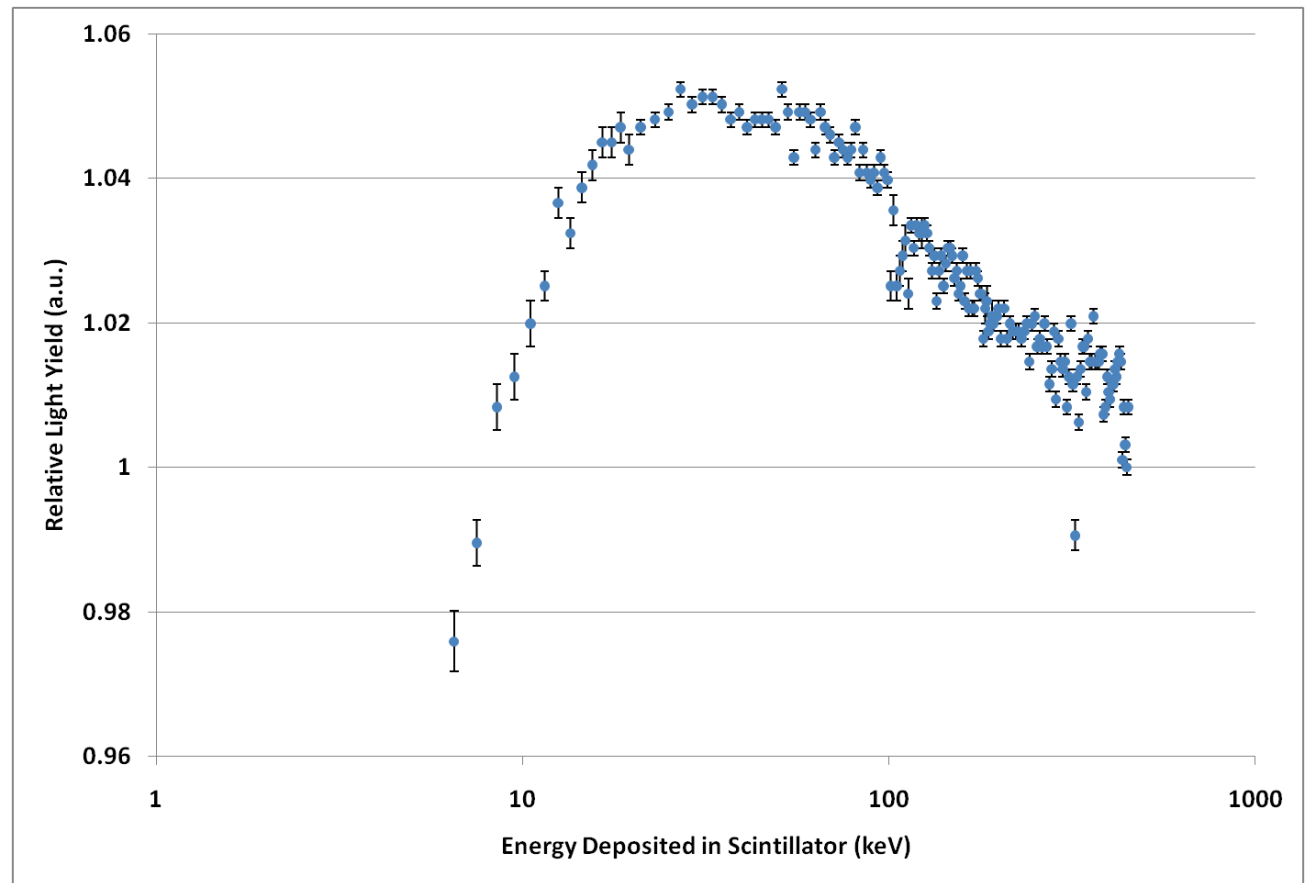

Figure 2. Relative light yield curve for $\mathrm{SrI}_{2}(\mathrm{Eu})$. The dopant level is $6 \%$ and the data has been renormalized to its value at $446 \mathrm{keV}$. 


\section{Lanthanum Bromide Data}

A study of the impact of cerium dopant level for $\mathrm{LaBr}_{3}$ scintillator on non-proportionality was also performed with the SLYNCI apparatus. Table 1 lists the characteristics of the crystals measured on SLYNCI. Except for the $0.5 \%$ cerium dopant level crystal (grown at RMD), all the crystals were manufactured by Saint Gobain and are marketed under the name "BrilLanCe 380". The 5\% cerium dopant level represents the standard crystal produced by Saint Gobain. Note that the light yield shows a decreasing trend with increasing cerium concentration. The light yield measurement for the 5\% dopant level crystal is based on what was seen in SLYNCI as opposed to a separate measurement. The relative light yield curves for all these crystals are shown in figure 3 . The curves are all very similar with the curves being the same at the few percent level, but note there is no significant rise in relative yield as the electron energy decrease from 446 $\mathrm{keV}$. There are also some significant systematic differences, again at higher energy. These differences trend with increasing dopant level with the $0.5 \% \mathrm{Ce}$ and $5 \%$ dopant level showing no difference.

\begin{tabular}{|c|c|c|c|}
\hline $\begin{array}{c}\text { Ce Dopant } \\
\text { Level }\end{array}$ & Manufacturer & Crystal Size & $\begin{array}{c}\text { Light Yield } \\
\text { photons/MeV) }\end{array}$ \\
\hline $0.5 \%$ & RMD & $\sim 10 \times 10 \times 10 \mathrm{~mm}$ & 77,000 \\
\hline $5.0 \%$ & St. Gobain & $10 \mathrm{~mm} \varnothing \times 10 \mathrm{~mm}$ & $\sim 70,000$ \\
\hline $10.0 \%$ & St. Gobain & $10 \mathrm{~mm} \varnothing \times 10 \mathrm{~mm}$ & 71,000 \\
\hline $20.0 \%$ & St. Gobain & $10 \mathrm{~mm} \varnothing \times 10 \mathrm{~mm}$ & 61,000 \\
\hline $30.0 \%$ & St. Gobain & $20 \mathrm{~mm} \varnothing \times 10 \mathrm{~mm}$ & 27,000 \\
\hline
\end{tabular}

Table 1. A list of the $\mathrm{LaBr}_{3}$ crystals used in the non-proportionality study.

\section{DISCUSSION}

To further interpret the $\mathrm{LaBr}_{3}$ data, we have fit the data the formulation described in reference [10]:

$\eta_{\mathrm{CAP}}=\left\{1-\eta_{\mathrm{ONS}} \exp \left[-(\mathrm{dE} / \mathrm{dx}) /(\mathrm{dE} / \mathrm{dx})_{\mathrm{ONS}}\right]\right\} /\left[1+(\mathrm{dE} / \mathrm{dx}) /(\mathrm{dE} / \mathrm{dx})_{\mathrm{BIRKS}}\right]$

which includes the formation of excitons from holes and electrons (maximum efficiency of $\eta_{\mathrm{ONS}}$ ) based on the so-called Onsager mechanism of Coulombic attraction, and incorporates the two stopping power parameters that describe the attraction between electrons and holes, $(\mathrm{dE} / \mathrm{dx})_{\mathrm{ONS}}$,

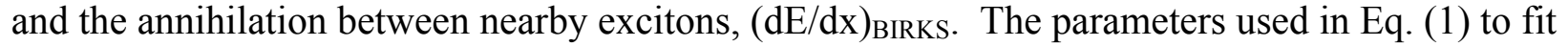
the data are compiled in Table 2, based on integrating Eq. (1) from the initial to final energies. We notice that $\eta_{\text {ONS }}$ value drops from $18.5 \%$ to $12 \%$ in passing from $0.5 \%$ Ce-doping to $30 \%$ doping. This means that less excitons are created (after the cascade) as the doping level is increased, probably because the carriers are more likely to be trapped at the Ce ions so that they are unable to recombine on a relevant timescale. We also notice that there is a slight increase in the Birks parameter $(\mathrm{dE} / \mathrm{dx})_{\mathrm{BIRKS}}$ indicating that exciton-exciton annihilation becomes less 
probable at high Ce doping. Lastly, the $(\mathrm{dE} / \mathrm{dx})_{\mathrm{ONS}}$ term is approximately constant as it is predicted to only be dependent on the host's dielectric constant.

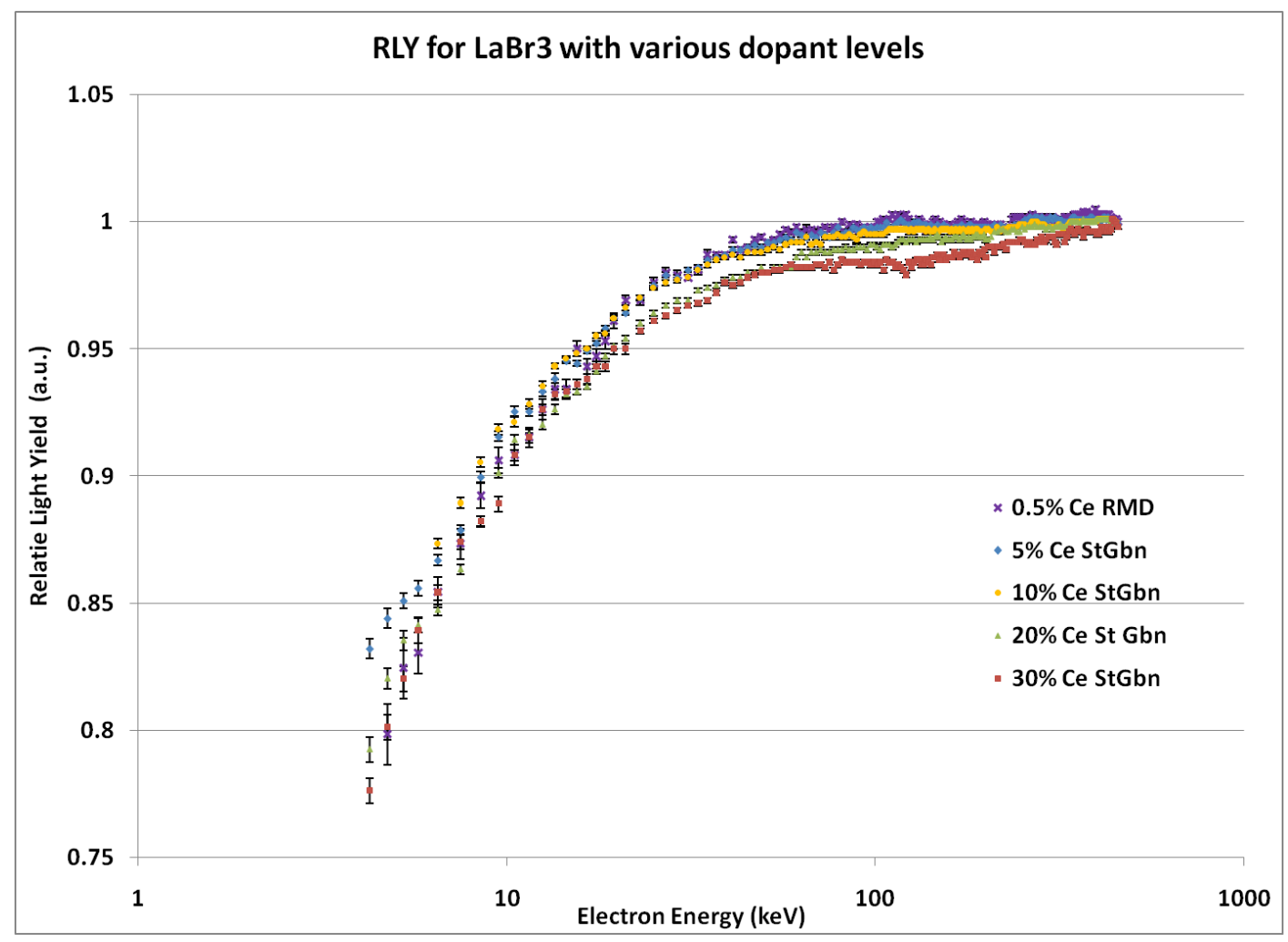

Figure 3. Relative light yield curves for $\mathrm{LaBr}_{3}$ with various $\mathrm{Ce}$ dopant levels.

\begin{tabular}{|c|c|c|c|}
\hline $\begin{array}{c}\mathrm{LaBr}_{3} \mathrm{Ce} \\
\text { doping level }\end{array}$ & $\begin{array}{c}(\mathrm{dE} / \mathrm{dx})_{\mathrm{ONS}} \\
(\mathrm{MeV} / \mathrm{cm})\end{array}$ & $\begin{array}{c}\mathrm{h}_{\mathrm{ONS}} \\
(\%)\end{array}$ & $\begin{array}{c}(\mathrm{dE} / \mathrm{dx})_{\mathrm{BIRKS}} \\
(\mathrm{MeV} / \mathrm{cm})\end{array}$ \\
\hline $0.5 \%$ & 36.4 & 18.5 & 370 \\
\hline $5 \%$ & 36.4 & 18 & 392 \\
\hline $10 \%$ & 36.4 & 15.5 & 444 \\
\hline $20 \%$ & 36.4 & 13 & 455 \\
\hline $30 \%$ & 36.4 & 12 & 455 \\
\hline
\end{tabular}

Table 2. Results of fits of the $\mathrm{LaBr}_{3}(\mathrm{Ce})$ non-proportionality curves to equation 1 .

\section{CONCLUSIONS}

Non-proportionality studies of various scintillators have been studied on SLYNCI. The non-proportionality of various alkali halides have been measured, showing similar behavior for all the crystals tested, with $\mathrm{NaI}$ showing a slightly better non-proportionality. $\mathrm{A} \mathrm{SrI}_{2}(\mathrm{Eu})$ crystal has also been tested which a similar shape but less non-proportionality. A series of $\mathrm{LaBr}_{3}$ crystals have also been tested, looking at the impact of Ce dopant level on non-proportionality. All the $\mathrm{LaBr}_{3}$ crystals showed very similar behavior with only small systematic difference seen 
with increasing dopant level. The studies of alkali halide and strontium halide are still a work in progress and more samples will be run in the near future.

\section{ACKNOWLEDGMENTS}

This work is supported by the National Nuclear Security Administration, Office of Defense Nuclear Nonproliferation, Office of Nonproliferation Research and Development (NA22) of the U.S. Department of Energy. Thanks to Eric Mattmann and Bruno Aleonard from St. Gobain for the loan of the $\mathrm{LaBr}_{3}(\mathrm{Ce})$, "BrilLanCe 380" crystals. This work performed under the auspices of the U.S. Department of Energy by Lawrence Livermore National Laboratory under Contract DE-AC52-07NA27344 and by Lawrence Berkeley National Laboratory under Contract No. DE-AC02-05CH11231.

\section{REFERENCES}

1. W.-S. Choong, K.M. Vetter, W.W. Moses, S.A. Payne, N.J. Cherepy, J.D. Valentine and G. Hull, "Design of a facility for measuring scintillator non-proportionality," IEEE Trans. Nucl. Sci., vol. 55, pp. 1753-1758, 2008.

2. W.-S. Choong, G. Hull, W.W. Moses, K.M. Vetter S.A. Payne, N.J. Cherepy, J.D. Valentine, "Performance of a Facility for Measuring Scintillator Non-Proportionality," IEEE Trans. Nucl. Sci., vol. 55, pp. 1073-1078, 2008.

3. G. Hull, S. Du, T. Niedermayr, S. Payne, N. Cherepy, A. Drobshoff, and L. Fabris "Light collection optimization in scintillator based gamma-ray spectrometers" Nucl. Instr. Meth. A, vol. 588, 384-388, 2008.

4. G. G. Kelley, P. R. Bell, R. C. Davis and N. H. Lazar, "Intrinsic scintillator resolution," IRE Transactions in Nuclear Science, vol. NS3, pp. 57-58, 1956.

5. C. L. Melcher, "Perspectives on the future of development of new scintillators," Nuclear Instruments and Methods in Physics Research A, vol. 537, pp. 6-14, 2005.

6. J. E. Jaffe, "Energy and length scales in scintillator nonproportionality," Nucl. Instr. \& Meth. A, vol. 580, 1378-1382 (2007); J. E. Jaffe, D. V. Jordan and A. J. Peurrung, "Energy nonlinearity in radiation detection materials: Causes and consequences," Nuclear Instruments and Methods in Physics Research A, vol. 570, pp. 72-83, 2007.

7. B.D. Rooney and J.D. Valentine, "Benchmarking the Compton Coincidence Technique for measuring electron response nonproportionality in inorganic scintillators ", IEEE Trans. Nucl. Sci., vol.43, no. 3, pp. 1271-1276, Jun. 1996.

8. T.D. Taulbee, B.D. Rooney, W. Mengesha, J.D. Valentine, "The measured electron response nonproportionalities of CaF2, BGO, and LSO", IEEE Trans. Nucl. Sci., vol. 44, no. 3, pp. 489493, Jun. 1997

9. W. Mengesha, T.D. Taulbee, B.D. Rooney, J.D. Valentine, "Light yield nonproportionality of CsI(Tl), CsI(Na), and YAP", IEEE Trans. Nucl. Sci., vol. 45, no. 3, pp. 456-461, Jun. 1998.

10. S. A. Payne, et al., IEEE Trans. Nucl. accepted for publication. 